\title{
Future development of the PLATO observatory for Antarctic science
}

\author{
Michael C. B. Ashley*a, Colin S. Bonner ${ }^{a}$, Jon R. Everett ${ }^{a}$, Jon S. Lawrence ${ }^{b, c}$, \\ Daniel Luong-van ${ }^{a}$, Scott $\operatorname{McDaid}^{a}$, Campbell McLaren ${ }^{a}$ and John W. V. Storey ${ }^{a}$ \\ ${ }^{a}$ School of Physics, University of New South Wales, Sydney NSW 2052, Australia ${ }^{b}$ Department \\ of Physics and Astronomy, Macquarie University, Sydney NSW 2109, Australia; \\ ${ }^{c}$ Australian Astronomical Observatory, Sydney NSW 1710, Australia;
}

\begin{abstract}
PLATO is a self-contained robotic observatory built into two 10-foot shipping containers. It has been successfully deployed at Dome A on the Antarctic plateau since January 2008, and has accumulated over 730 days of uptime at the time of writing. PLATO provides $0.5-1 \mathrm{~kW}$ of continuous electrical power for a year from diesel engines running on Jet-A1, supplemented during the summertime with solar panels. One of the 10-foot shipping containers houses the power system and fuel, the other provides a warm environment for instruments. Two Iridium satellite modems allow $45 \mathrm{MB}$ /day of data to be transferred across the internet.

Future enhancements to PLATO, currently in development, include a more modular design, using lithium iron-phosphate batteries, higher power output, and a light-weight low-power version for field deployment from a Twin Otter aircraft.

Technologies used in PLATO include a CAN (Controller Area Network) bus, high-reliability PC/104 computers, ultracapacitors for starting the engines, and fault-tolerant redundant design.
\end{abstract}

Keywords: PLATO, Antarctica, robotic observatory

\section{INTRODUCTION}

The Antarctic plateau, by virtue of its high elevation, coldness, still atmosphere, and low precipitable water vapour, is likely to contain the best astronomical observatory sites on the surface of the earth. ${ }^{1,2}$ There are only two permanently occupied stations on the plateau: South Pole and Dome C. In order to conduct experiments away from these stations, a solution is needed to provide power, heat, and communications. This is particularly challenging since the best time for optical astronomy is mid-winter, which is five months after the last possible human contact with the experiments, and when solar power is no longer available.

The PLATO (PLATeau Observatory) ${ }^{3,4}$ was created to solve this problem. PLATO provides between 0.5$1 \mathrm{~kW}$ of continuous electrical power for a year, and a warm environment for instruments. Power comes from diesel engines running on 4000 liters of Jet-A1, supplemented by solar panels during the summer months. ${ }^{5}$ Communications, for control, health and status information, and limited return of scientific data, is provided by two Iridium satellite modems.

PLATO was deployed by a Chinese traverse to Dome A, the highest point on the Antarctic plateau, in January 2008 (Figure 1). It ran for 204 days continuously during the first year, and, following a servicing mission in January 2009, has been operating non-stop (526 days at the time of writing in June 2010). PLATO has supported a variety of astronomical experiments, including wide-field optical imaging telescopes $\left(\mathrm{CSTAR}^{6}\right)$, widefield sky imagers $\left(\right.$ Gattini $\left.^{7}\right)$, terahertz telescopes (Pre-HEAT ${ }^{8,9}$ ), a $0.75-15 \mathrm{THz}$ fourier-transform spectrometer, and sonic radars to probe the turbulence in the atmosphere (Snodar ${ }^{10}$ ). Roughly 10 GB of data have been returned via Iridium, with several TB being retrieved on hard disks during subsequent traverse expeditions undertaken by the Polar Research Institute of China.

The success of PLATO at Dome A has led to plans to deploy an additional power module at Dome A, and to build a new PLATO for Dome F. There are also plans for lightweight, low-power $(\sim 200 \mathrm{~W})$ versions. This paper discusses some of the design improvements being made to PLATO as we build two new versions during 2010 at UNSW.

\footnotetext{
*m.ashley@unsw.edu.au
} 


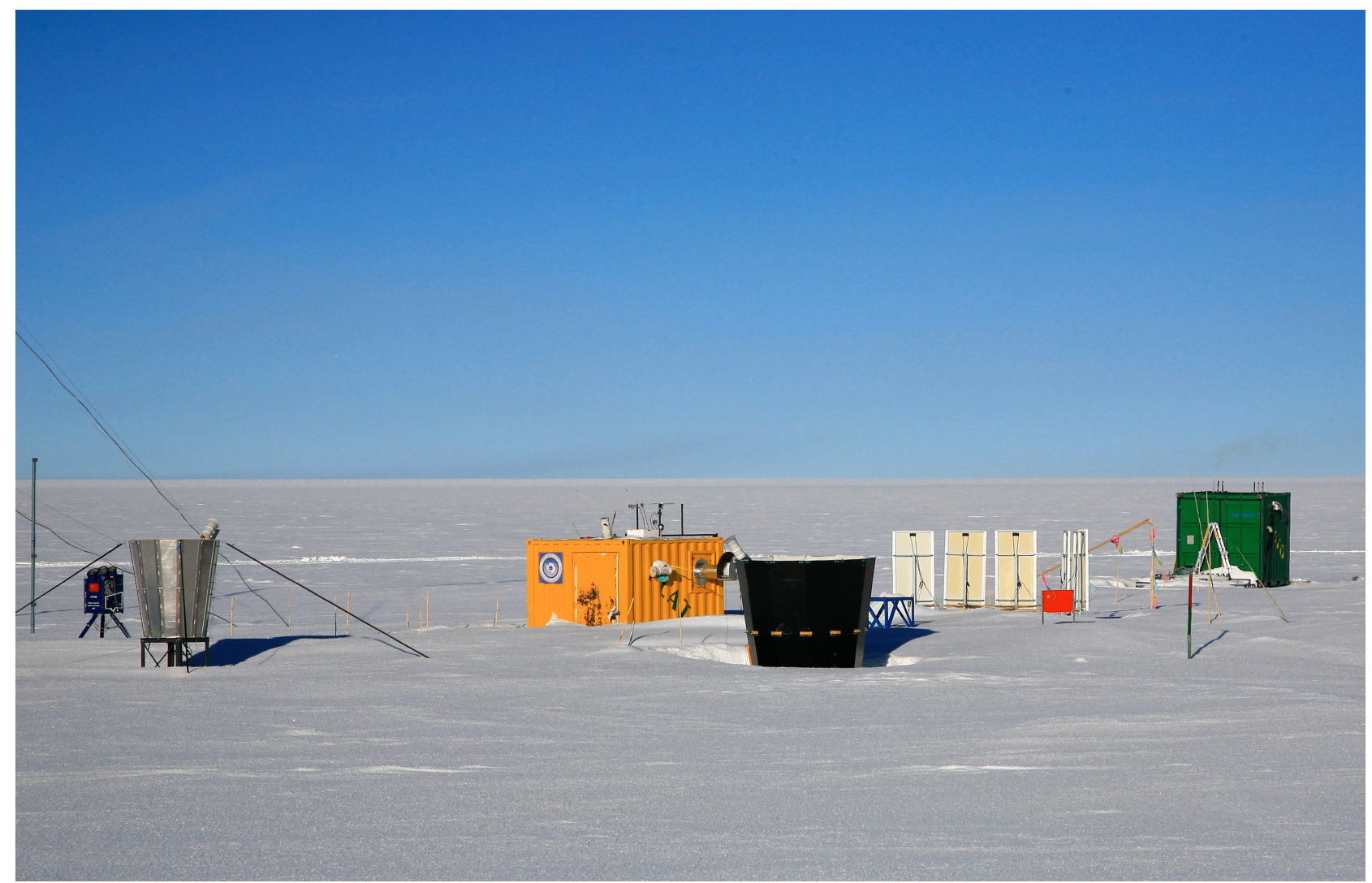

Figure 1. The PLATO remote observatory at Dome A, Antarctica, in January 2010. The Instrument Module is yellow (to the left of center), the Engine Module is green (to the right). They are separated by $\sim 50 \mathrm{~m}$, which appears foreshortened in this photograph. Three of the six solar panels are clearly visible. The two truncated cones are Snodar sonic radars. The CSTAR telescopes are visible to the left of the leftmost Snodar. A variety of other instruments are installed on the roof of the Instrument Module. Image credit: Z. Shang.

\section{DESIGN IMPROVEMENTS}

\subsection{Batteries}

Diesel engines are more reliable if run hard, ensuring that the exhaust temperatures are high and no unburned fuel clogs the exhaust system. This requirement naturally leads to the need for a large amount of electrical power stored in batteries so that the diesel engines can be run hard and intermittently as required.

PLATO currently uses $230 \mathrm{~kg}$ of sealed lead-acid (SLA) batteries (Sungel 4SG320) providing $6 \mathrm{kWHr}$ of electrical power at a nominal $24 \mathrm{~V}$. SLA batteries have the advantage of retaining some usable capacity down to temperatures as low as $-40^{\circ} \mathrm{C}$, but have limited ability to withstand deep cycling. In practice, this means that only a fraction, perhaps $30 \%$, of the rated capacity is used. SLAs also have a high-temperature coefficient of voltage, and a relatively low maximum charge/discharge rate.

The new PLATO design uses $270 \mathrm{~kg}$ of Thundersky lithium iron phosphate (LiFePO4) batteries, providing $22 \mathrm{kWHr}$ of electrical power at $120 \mathrm{~V}$. LiFePO4 has the disadvantage of only operating down to $-25^{\circ} \mathrm{C}$, however, it possesses the considerable advantages of being amenable to deep cycling, having a low temperature coefficient, and a high charge/discharge rate. For these reasons, LiFePO4 should be a better choice for our application, and will allow the diesel engines to be operated efficiently. One issue with LiFePO4 is that it is of critical importance to balance the voltage of the cells in the battery pack, and to guarantee that no individual cell ever over-charges. To satisfy this requirement we will be using cell monitoring and control modules made by Tritium. 
These modules communicate using a CAN bus, and will allow the individual monitoring of the health and status of every cell.

\subsection{Electrical bus}

The original PLATO design used a 24VDC power bus, with a 150VDC cable joining the Instrument and Engine Modules, which are separated by $50 \mathrm{~m}$. With the requirement to support higher power instruments, a 24VDC bus is not an efficient choice. This has led us to standardise on a 120VDC bus directly connected to the LiFePO4 battery pack, as shown in Figure 2. A low-power 24VDC bus will be provided for legacy instruments, and $230 \mathrm{VAC} / 50 \mathrm{~Hz}$ will be generated for transmission to higher power instruments.

One issue with a high-voltage DC bus is the safety of human operators. To maximise safety, all the buses (120VDC, 24VDC, and CAN power) will be floating, with high impedance connections to chassis ground. The voltage drops across these high impedance connections will be monitored, and fault conditions will lead to automatic disconnection of the power.

The new PLATO bus design will allow us to relatively easily add additional Engine Modules in parallel to increase the power capacity.

An alternative to a high-voltage DC bus would be a 230VAC micro-grid system. This has a number of advantages, including the easy addition of power sources such as solar panels and wind generators using commercial off-the-shelf grid-feed systems.

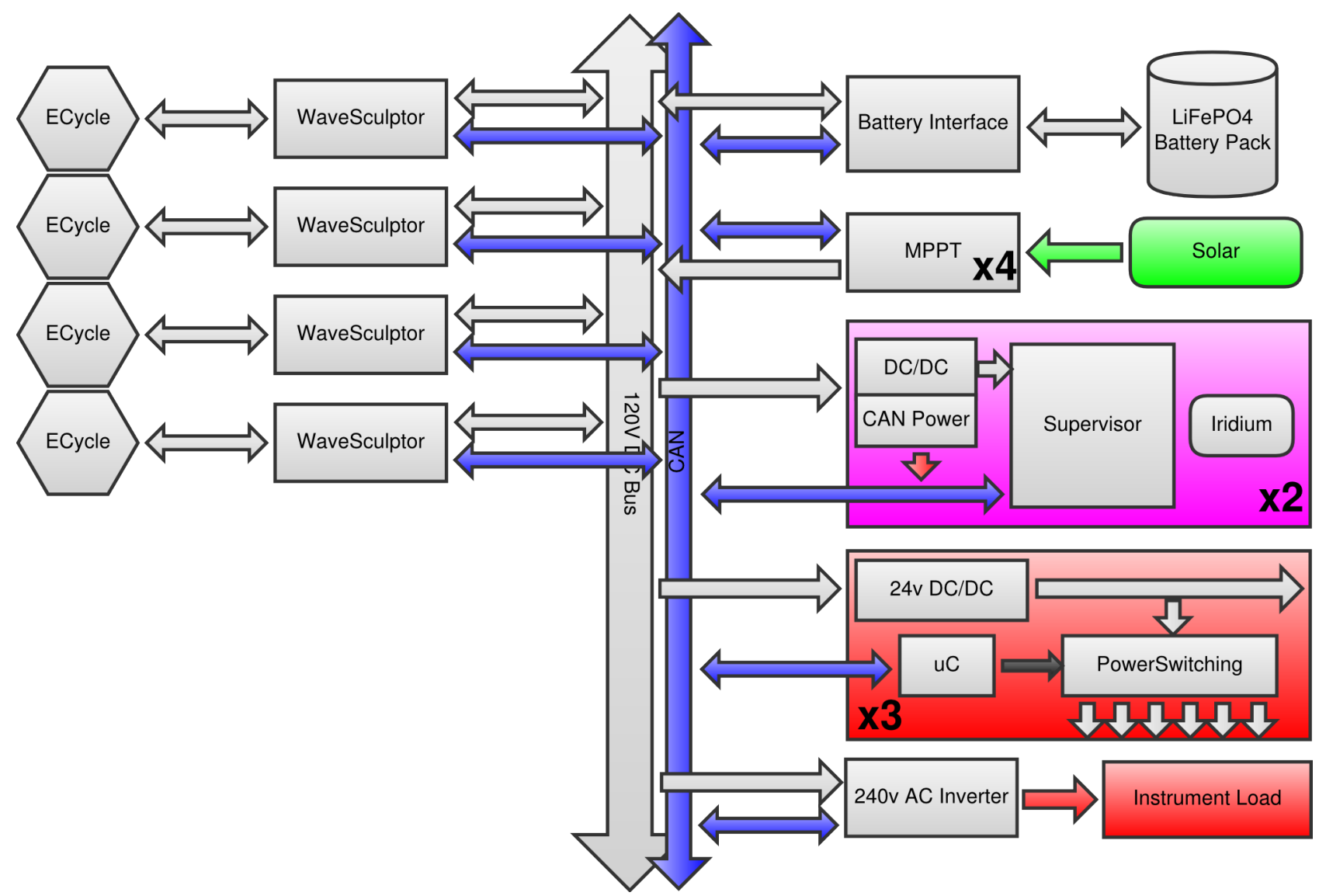

Figure 2. A schematic diagram of the power generation and distribution components within the new PLATO design. The left hand side is the Engine Module, the right hand side is the Instrument Module. 


\subsection{Engine control}

In the existing PLATO, the diesel engines are started by 12VDC starter motors, powered from banks of ultracapacitors - chosen for excellent high current low temperature performance. ${ }^{11}$ Each engine crankshaft directly drives a high efficiency three-phase alternator, the output of which is then rectified to produce the 150VDC for the Instrument Module. Figure 3 shows a view inside the Engine Module.

In the new design we will be using the alternators to start the engines. This has the advantage of eliminating the need for starter motors, starter solenoids, ultracapacitors, and the associated high current wiring. The engine starting and DC power generation will be handled by a WaveSculptor from Tritium. This unit is CAN-controlled, and gives great flexibility for engine management. For example, the WaveSculptor allows measurement of input and output voltage/current, engine RPM, and the ability to control the engine load. The WaveSculptor is capable of "sensor-less start", i.e., it does not need to know the initial position of the alternator stator -it establishes this position by driving the alternator with sufficient current to force the stator to follow, and then, when a high enough angular velocity is reached, it can determine the stator position from the back EMF.

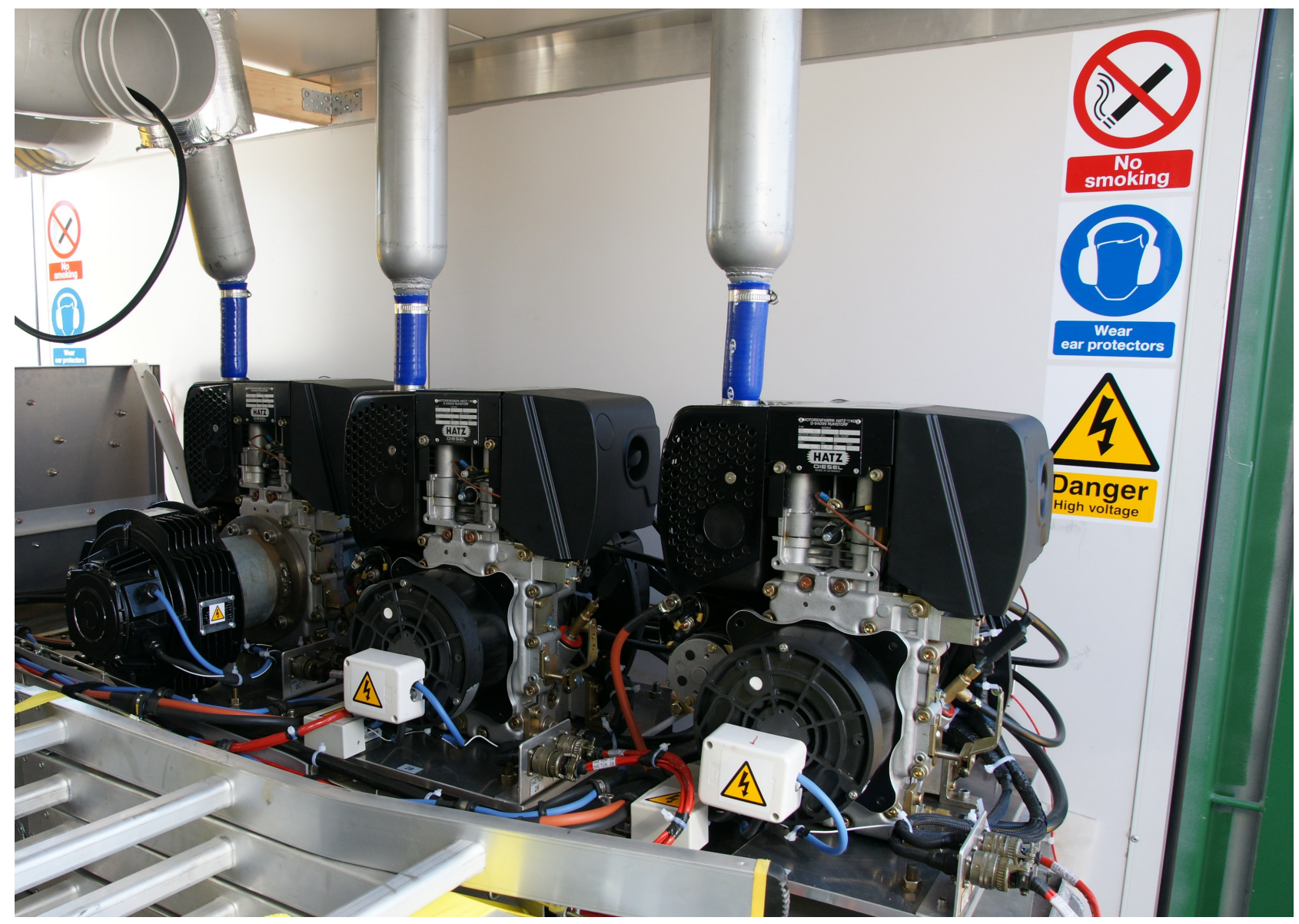

Figure 3. A view inside the PLATO Engine Module, prior to the original deployment in January 2008. Three of the six Hatz diesel engines are visible. The new design will place the exhaust tubes out the side rather than the top. The high-current starter motor wiring will be eliminated. The engines will be on modular, easily-replaceable, plates.

\subsection{Supervisor computer}

The original PLATO uses a pair of redundant Parvus CPU-1452 PC/104 computers running Debian Lenny with a read-only file system. ${ }^{12} \mathrm{~A}$ watchdog timer resets the computer under fault conditions. These computers have been absolutely reliable, with the only issue being occasionally running out of memory due to the limit of $256 \mathrm{MB}$ 
shared between running processes and temporary file systems. The relatively slow CPU also limits the use of real-time data analysis.

Data storage is provided by four 2.5-inch hard disks with capacities of 500 to $750 \mathrm{~GB}$ each. The 2.5-inch format has the advantage that a single 5VDC power supply needed. The disks are connected to the computer via USB. SATA would probably be preferable, as USB interfaces for hard disks can have firmware issues that affect their reliability.

For the new design we will be using a Parvus ISIS-XL PC/104 system, with $2 \mathrm{~GB}$ of memory and a $1.3 \mathrm{GHz}$ processor. We will continue to use the 2.5-inch hard disk solution. Solid-state disks are also a possibility, although, in our experience, they are more susceptible to catastrophic total data loss if written to when cold.

\subsection{Miscellaneous improvements}

In addition to the changes detailed above, the new PLATO design will be lighter, by virtue of using fibreglass rather than steel, and more easily transportable by helicopter - it is designed to be relatively simple to disassemble into 1 tonne components with limited wind loading.

A major consideration has been improving the serviceability of the Engine Module. A removal roof will allow convenient access to the engines without having to stoop.

Other miscellaneous design changes include a fully optically isolated CAN bus, and the use of teflon fuel and oil hoses. We are also increasing the fuel tank capacity from 4000 liters to 6000 liters, both to provide more power for the Dome A version, and to give the option of running for two years without servicing at reduced power for the Dome $\mathrm{F}$ version.

\subsection{Low-power version}

Some experiments in Antarctica have a requirement for modest $(\sim 200 \mathrm{~W})$ continuous electrical power throughout a year. We are currently in the early design stages of modifying the PLATO concept for this application, with the aim of building a module that could be deployed by Twin Otter aircraft.

\section{CONCLUSION}

We have outlined in this paper a number of improvements that we will be making to the PLATO design during 2010. It is always risky to change a system that has proven its reliability, however, we are driven to make the evolutionary changes described herein by the higher power requirements of future experiments, and considerations of the ease of serviceability and deployment.

\section{ACKNOWLEDGMENTS}

This research is financially supported by the Australian Research Council, the Australian Antarctic Division, and the University of New South Wales. The operation of PLATO at Dome A has been supported by the Chinese PANDA International Polar Year project and the Polar Research Institute of China. Additional support has been provided by the US National Science Foundation, and the United States Antarctic Program.

\section{REFERENCES}

[1] Saunders, W., Lawrence, J. S., Storey, J. W. V., Ashley, M. C. B., Kato, S., Minnis, P., Winker, D., Guiping Liu, and Kulesa, C. "Where is the best site on Earth? Domes A, B, C and F, and Ridges A and B", Publ. Astron. Soc. Pac., 121, 976-992 (2009).

[2] Lawrence, J. S., Ashley, M. C. B., Tokovinin, A. and Travouillon, T., "Exceptional astronomical seeing conditions above Dome C in Antarctica," Nature, 431, 278-281 (2004).

[3] Lawrence, J. S., Allen, G. R., Ashley, M. C. B., Bonner, C., Bradley, S., Cui, X., Everett, J. R., Feng, L., Gong, X., Hengst, S., Hu, J., Jiang, Z., Kulesa, C. A., Li, Y., Luong-Van, D., Moore, A. M., Pennypacker, C., Qin, W., Riddle, R., Shang, Z., Storey, J. W. V., Sun, B., Suntzeff, N., Tothill, N. F. H., Travouillon, T., Walker, C. K., Wang, L., Yan, J., Yang, J., Yang, H., York, D., Yuan, X., Zhang, X. G., Zhang, Z., Zhou, X., and Zhu, Z., "The PLATO Antarctic site testing observatory", Proc. SPIE, 7012, 701227-1-701227-12 (2008). 
[4] Yang, H., Allen, G., Ashley, M. C. B., Bonner, C. S., Bradley, S., Cui, X., Everett, J. R., Feng, L., Gong, X., Hengst, S., Hu, J., Jiang, Z., Kulesa, C. A., Lawrence, J. S., Li, Y., Luong-Van, D., McCaughrean, M. J., Moore, A. M., Pennypacker, C., Qin, W., Riddle, R., Shang, Z., Storey, J. W. V., Sun, B., Suntzeff, N., Tothill, N. F. H., Travouillon, T., Walker, C. K., Wang, L., Yan, J., Yang, J., York, D., Yuan, X., Zhang, X., Zhang, Z., Zhou, X. and Zhu, Z., "The PLATO Dome A Site-Testing Observatory: Instrumentation and First Results", Publ. Astron. Soc. Pac., 121, 174-184 (2009).

[5] Hengst, S., Allen, G. R., Ashley, M. C. B., Everett, J. R., Lawrence, J. S., Luong-Van, D. M., and Storey, J. W. V., "PLATO power: a robust low environmental impact power generation system for the Antarctic plateau", Proc. SPIE, 7012, 70124E-1-70124E-10 (2008).

[6] Zhou, X., Fan, Z., Jiang, Z., Ashley, M. C. B., Cui, X., Feng, L., Gong, X., Hu, J., Kulesa, C. A., Lawrence, J. S., Liu, G., Luong-Van, D. M., Ma, J., Moore, A. M., Qin, W., Shang, Z., Storey, J. W. V., Sun, B., Travouillon, T., Walker, C. K., Wang, J., Wang, L., Wu, J., Wu, Z., Xia, L., Yan, J., Yang, J., Yang, H., Yuan, X., York, D., Zhang, Z., and Zhu, Z., "The First Release of the CSTAR Point Source Catalog from Dome A, Antarctica", Publ. Astron. Soc. Pac., 122, 347-353 (2010).

[7] Moore, A., et al., "Gattini: a multisite campaign for the measurement of sky brightness in Antarctica", Proc. SPIE, 7012, 701226-1-701226-10 (2008).

[8] Kulesa, C. A., Walker, C. K., Schein, M., Golish, D., Tothill, N., Siegel, P., Weinreb, S., Jones, G., Bardin, J., Jacobs, K., Martin, C. L., Storey, J., Ashley, M., Lawrence, J., Luong-Van, D., Everett, J., Wang, L., Feng, L., Zhu, Z., Yan, J., Yang, J., Zhang, X.-G., Cui, X., Yuan, X., Hu, J., Xu, Z., Jiang, Z., Yang, H., Li, Y., Sun, B., Qin, W., and Shang, Z., "Pre-HEAT: submillimeter site testing and astronomical spectra from Dome A, Antarctica", Proc. SPIE, 7012, 701249-1-701249-11 (2008).

[9] Yang, H., Kulesa, C. A., Walker, C. K., Tothill, N. F. H., Yang, J., Ashley, M. C. B., Cue, X., Feng, L., Lawrence, J. S., Luong-Van, D. M., Storey, J. W. V., Wang, L., Zhou, X., and Zhu, Z., "Exceptional terahertz transparency and stability above Dome A, Antarctica", Publ. Astron. Soc. Pac., 122, 490-494 (2010).

[10] Bonner, C. S., Ashley, M. C. B., Lawrence, J. S., Luong-Van, D. M., and Storey, J. W. V., "Snodar: An acoustic radar for atmospheric turbulence profiling with $1 \mathrm{~m}$ resolution", Acoustics Australia, 37, 47-51 (2009).

[11] Hengst, S., Luong-Van, D. M., Everett, J. R., Lawrence, J. S., Ashley, M. C. B., Castel, D., and Storey, J. W. V., 2010, "A small, high-efficiency diesel generator for high-altitude use in Antarctica", International Journal of Energy Research, DOI: 10.1002/er.1595 (2009).

[12] Luong-Van, D. M., Ashley, M. C. B., Everett, J. R., Lawrence, J. S., and Storey, J. W. V., "PLATO control and robotics", Proc. SPIE, 7019, 70192U-1-70192U-10 (2008). 